\title{
ONYGENALES (EUROTIOMYCETES, ASCOMYCOTA) QUERATINOFILICOS EN SUELOS DE ESTABLECIMIENTOS EDUCACIONALES URBANOS Y RURALES DE LA V REGION, CHILE.
}

\author{
Queratinophilic Onygenales (Eurotiomycetes, Ascomycota) in urban and rural \\ soils of eduacational center of V Region, Chile)
}

\author{
Toro, M.A., Ferrari, B., Pino, J., \& Piontelli, E. \\ Universidad de Valparaíso, Escuela de Medicina, Cátedra de Micología, \\ Casilla 93 V, Valparaíso, Chile. email: maria.alicia.toro@vtr.net
}

Palabras clave: Onygenales, hongos queratinofílicos, suelos urbanos-rurales, establecimientos educacionales. Key words: Onygenales, keratinophilic fungi, urbans-rural soils, educacional centers.

\section{RESUMEN}

Con la finalidad de estudiar la presencia de Onygenales queratinofílicas potencialmente patógenas para el hombre y los animales, se analizaron mediante la técnica del anzuelo queratínico (Marzo - Diciembre, 2006), suelos de establecimientos educacionales urbanos y rurales de la V Región, Chile. Se colectaron un total de 64 muestras, de las cuales, la mitad se obtuvieron en Valparaíso-Viña del Mar (urbano) y la otra en Olmué-Limache (en zonas rurales). En zona urbana se aislaron 112 cepas (7 géneros y 12 especies) y en la rural 147 (11 géneros y 18 especies). Los géneros de mayor prevalencia en la zona urbana y rural en orden decreciente fueron: Chrysosporium y su teleomorfo $32 \%$ versus 45\%; Keratinomyces y su teleomorfo, $26 \%$ y $16 \%$; Microsporum y su teleomorfo $23 \%$ y $16 \%$ y Myceliophthora y su teleomorfo con un 13 y 10\%. Amauroascus mutatus, Auxarthron umbrinum, Gymnoascus reessii, Chrysosporium charmichaelii, Ch. merdarium, Ch. tropicum, Geomyces pannorum var. pannorum fueron detectados sólo en la zona rural; mientras que Malbranchea flava sólo en la zona urbana. Mediante la técnica de Takashio, se pudo identificar separadamente las especies del complex Microsporum gypseum, determinándose que M. gypseum (Arthrodema gypseum) y M.fulvum (A. fulvum) obtuvieron una frecuencia de aislamiento similar en ambas zonas, siendo el primero un oportunista potencialmente patógeno para el hombre y los animales.

Recibido el 16 de Agosto 2007

Aceptado el 12 de Diciembre 2007

\section{ABSTRACT}

With the purpose of studying the presence of keratinophilic Onygenales that are potentially pathogenous for man and animal, urban and rural soils from educational centers in the VRegion were examined with the keratinic bait technique (march-december 2006).

A total of 64 samples were collected, 32 of them being from Valparaíso-Viña del Mar (urban) while the rest in Olmué-Limache (in rural zones). One hundred and twelve strains ( 7 genera and 12 species) were collected in the urban zone and 147 strains in the rural zone (11 genera and 18 species). Genera with the highest prevalence both in the urban and the rural zones were, in decreasing order: Chrysosporium and its teleomorph $32 \%$ vs. $45 \%$; Keratinomyces and its teleomorph $26 \%$ and $16 \%$; Microsporum and its teleomorph $23 \%$ and $16 \%$ and Myceliophthora and its. teleomorph with 13\% and $10 \%$. Amauroascus mutatus, Auxarthron umbrinum, Gymnoascus reessii, Chrysosporium charmichaelii, Ch. merdarium, Ch.tropicum, Geomyces pannorum var. pannorum were detected only in the rural zone, whereas Malbranchea flava only in the urban zone.

Species of the complex Microsporum gypseum, could be identified separately by means of the Takashio technique, coming to the conclusion that M. gypseum (Arthrodema gypseum) and M.fulvum (A. fulvum) achieved a similar frequency of isolation in both zones and that the former is a potentially opportunistic pathogen for man and animal. 


\section{INTRODUCCION}

Numerosos investigadores han señalado que la distribución de los hongos queratinofílicos pertenecientes a los Onygenales, está condicionada por el contenido orgánico de los suelos y en particular por la presencia de substratos queratínicos procedentes del hombre y otros mamíferos. Esta situación constituye un importante reservorio de hongos potencialmente patógenos (English, 1965; Dominik et al., 1973; Marchisio, 1986, Piontelli \& Toro, 1987; Caretta et al., 1989; De Hoog et al., 2000).

Los estudios realizados en diversas partes del mundo analizando las condiciones naturales de diferentes hábitat abundantes en queratina, especialmente el suelo de áreas agrícolas, jardines, playas recreacionales, parques y colegios, confirman la amplia presencia de estos hongos. (Alvarez \& Bracalenti 1984; Piontelli et al. 1984; Armes \& Hilda, 1998; Mangiaterra et al., 2000; Shadzi et al., 2002; Vidyasagar et al., 2005). Los suelos de los establecimientos educacionales y áreas circundantes, son frecuentemente fuente de substratos queratínicos aportados por animales domésticos, aves y presencia humana (estudiantes), presentan gran interés ecológico y epidemiológico (Marchisio et al., 1986; Armes \& Hilda, 1998).

La metodología de estudio de estos hongos especializados nutricionalmente se inició en 1952 por Vanbreuseghem, seguidos por Ajello (1960), dos científicos que marcaron un hito en su estudio, implementando el primero la técnica del anzuelo queratínico, modificada posteriormente por Orr (1969).

En la presente investigación, se analizó la micota queratinofílica en suelos de establecimientos educacionales en zonas urbanas y rurales, para determinar la presencia de Onygenales saprófitas y potencialmente patógenas, en estos hábitat, durante el período Marzo a Diciembre de 2006.

\section{MATERIALES Y METODOS}

Durante el período Marzo a Diciembre del 2006, se procesaron los suelos de 16 establecimientos educacionales de la V Región (Chile), 8 correspondientes a zonas urbanas (Valparaíso-Viña del Mar) y 8 a zonas rurales (Olmué-Limache), con un total de 32 muestras por cada zona. Estas fueron recolectadas en 4 puntos por establecimiento, en forma aleatoria, en superficies de aproximadamente $50 \mathrm{~m}^{2}$, en 4 bolsas estériles en una cantidad aproximada de 200 gramos y a una profundidad de 1 a 3 $\mathrm{cm}$. Este material fue congelado a $-14^{\circ} \mathrm{C}$ por 24 horas antes de ser procesado en el laboratorio, a fin de prevenir la proliferación de ácaros. Posteriormente, se distribuyó en duplicado en 4 placas. A éstas se les adicionó en superficie el pelo estéril de niños prepuber de 1-2 cm de largo manteniéndolas humectadas con una solución de agua estéril con cloranfenicol $(0,25 \mathrm{~g} / \mathrm{L})$, para controlar el crecimiento bacteriano. Las placas fueron mantenidas durante la primera semana de incubación a $25^{\circ} \mathrm{C}$ y posteriormente se mantuvieron a temperatura ambiente durante 1 mes. La revisión del material inoculado se realizó mediante observación macroscópica con lupa estereoscópica para detectar el crecimiento y el posterior aislamiento entre los 20 a 30 días. Esta observación fue complementada con microscopía, mediante la técnica de observación al fresco con azul de algodón. Los cultivos y subcultivos se realizaron en los medios PYE (agar, phytona, extracto de levadura) adicionado con Dichloran $(0,00025 \mathrm{~g} / \mathrm{L})$ para el género Chrysosporium y agar Takashio (1973) para identificar y diferenciar el complex Microsporum gypseum según lo descrito por Demage et al., (1992). Las especies aisladas de éste género fueron inoculadas a 27 y $37^{\circ} \mathrm{C}$, para separar las potencialmente patógenas (crecimiento a $37^{\circ} \mathrm{C}$ ). El resto de las especies aisladas fueron sembradas e identificadas ya sea directamente sobre el substrato queratínico o en agar papa dextrosa. La identificación de los Onygenales se llevo a cabo utilizando principalmente las claves taxonómicas de los siguientes autores Charmichael, 1962, Rebell \& Taplin, 1970, Cano \& Guarro, 1990, van Oorschot, 1980, Domsch et al., 1980, Currah, 1985, Demange et al., 1992, entre otras.

Cada taxa se contabilizó una sola vez en cada muestra, no importando si se repetía en la misma placa o en el duplicado.

Se aplicó el índice de Shannon -Wiever para comparar la diversidad de especies en ambas zonas.y el indice de Jaccard para la similitud.

\section{RESULTADOS}

Por medio de la técnica del anzuelo queratinofílico se aislaron 11 géneros de Onygenales, con mayor diversidad en zonas rurales (9 géneros) y 6 en urbanas. Los géneros con mayor cantidad de especies fueron Chrysosporium (7) y Microsporum (3) incluyendo los teleomorfos correspondientes, este último, con las mismas especies en ambas zonas pero con diferencias en la frecuencia de presencia (Tabla 1). Los mayores porcentajes de frecuencia en zona urbana fueron para Chrysosporium (32\%), Keratinomyces (26\%), Microsporum (23\%) y Myceliophthora (13\%), mientras en zona rural: Chrysosporium (45\%), Keratinomyces (16\%), Microsporum (16\%) y Myceliophthora (10\%). Se incluyen en estos géneros los teleomorfos correspondientes (Tabla 1, Gráfico 1).

La Frecuencia de presencia de taxa de Onygenales por zona en los establecimientos educacionales analizados, es mayor en la zona rural, excepto en los establecimientos 
Tabla 1 Frecuencia de Taxa Onygenales aisladas en los 8 colegios en zonas urbanas y rurales

\begin{tabular}{|c|c|c|c|c|c|c|c|c|c|c|c|c|c|c|c|c|c|c|c|c|}
\hline \multirow[t]{2}{*}{ Taxa* } & \multicolumn{10}{|c|}{ Urbano } & \multicolumn{10}{|c|}{ Rural } \\
\hline & 1 & 2 & 3 & 4 & 5 & 6 & 7 & 8 & Total & $\%$ & 1 & 2 & 3 & 4 & 5 & 6 & 7 & 8 & Total & $\%$ \\
\hline Amauroascus mutatus (Quelet) Rameeloo & & & & & & & & & 0 & 0,0 & 1 & 1 & 1 & 2 & & & & & 5 & 3,4 \\
\hline Aphanoascus sp. & & & 1 & 1 & & & & & 2 & 1,8 & & & & & & & 2 & & 2 & 1,4 \\
\hline $\begin{array}{l}\text { Auxarthron umbrinum (Boud.) Orr \& } \\
\text { Plunkett }\end{array}$ & & & & & & & & & 0 & 0,0 & 2 & & & & & & & & 2 & 1,4 \\
\hline \begin{tabular}{|l} 
Chrysosporium carmichaelii van \\
Oorschot
\end{tabular} & & & & & & & & & 0 & 0,0 & & & 1 & 3 & & 2 & & 1 & 7 & 4,8 \\
\hline $\begin{array}{l}\text { Chrysosporium indicum Randhawa } \\
\& \text { Sanhu }\end{array}$ & 1 & & & 1 & & & 1 & & 3 & 2,7 & 1 & 2 & & 2 & & & 2 & 1 & 8 & 5,4 \\
\hline $\begin{array}{l}\text { Chrysosporium keratinophilum D. Frey } \\
\text { ex Charmichael y su teleomorfo Aphano- } \\
\text { ascus Keratinophilus Punsola \& Cano } \\
\end{array}$ & 1 & 1 & & 3 & 2 & 1 & 1 & & 9 & 8,0 & 1 & 2 & 1 & 2 & & 2 & 4 & 1 & 13 & 8,8 \\
\hline $\begin{array}{l}\text { Chrysosporium merdarium (Link ex } \\
\text { Grev.) Carmichael }\end{array}$ & & & & & & & & & 0 & 0,0 & 1 & & & & & & & & 1 & 0,7 \\
\hline $\begin{array}{l}\text { Chrysosporium pannicola (Corda) van } \\
\text { Oorschot \& Stalpers } \\
\end{array}$ & 3 & 3 & 1 & 2 & 1 & 1 & 2 & & 13 & 11,6 & 1 & & 2 & 2 & 2 & 4 & 4 & & 15 & 10,2 \\
\hline Chrysosporium tropicum Carmichael & & & & & & & & & 0 & 0,0 & & & 2 & 1 & & & & & 3 & 2,0 \\
\hline $\begin{array}{l}\text { Chrysosporium sp. y su teleomorfo } \\
\text { Aphanoascus fulvescens Cooke }\end{array}$ & 1 & 1 & 1 & 2 & 2 & 1 & 1 & & 9 & 8,0 & 2 & & 2 & 4 & & 3 & 3 & 3 & 17 & 11,6 \\
\hline $\begin{array}{l}\text { Geomyces pannorum (Link) Sigler \& } \\
\text { Carmichael }\end{array}$ & & & & & & & & & 0 & 0,0 & 3 & & & & 1 & & 1 & & 5 & 3,4 \\
\hline Gymnoascus reessii Baran. & & & & & & & & & 0 & 0,0 & 1 & & & 1 & & & & & 2 & 1,4 \\
\hline $\begin{array}{l}\text { Keratinomyces ajelloi Vanbreuseghem y } \\
\text { su teleomorfo Arthrderma uncinatum } \\
\text { Dawson \& Gentles } \\
\end{array}$ & 4 & 3 & 3 & 4 & 4 & 4 & 4 & $\mathbf{3}$ & 29 & 25,9 & 3 & 4 & 4 & 4 & 3 & 2 & 2 & 1 & 23 & 15,6 \\
\hline Malbranchea flava Sigler \& Carmichael & & & & 1 & & & & 1 & 2 & 1,8 & & & & & & & & & 0 & 0,0 \\
\hline Microsporum cookei Ajello & & 1 & & & & & 2 & & 3 & 2,7 & & & & & & & 1 & & 1 & 0,7 \\
\hline $\begin{array}{l}\text { Microsporum fulvum Uriburu y su } \\
\text { teleomorfo Arthroderma fulvum } \\
\text { (Stockdale) Weitzman et al. }\end{array}$ & & 1 & 2 & 2 & 1 & 1 & 2 & 1 & 10 & 8,9 & 2 & 1 & 2 & 2 & & 1 & 1 & & 9 & 6,1 \\
\hline $\begin{array}{l}\text { Microsporum gypseum (Bodin) Guiart \& } \\
\text { Grigorakis y su teleomorfo Arthroder- } \text { ma } \\
\text { gupseum (Nannizzi) Weitzman et al. }\end{array}$ & 4 & 2 & & 1 & 2 & 1 & 1 & 2 & 13 & 11,6 & 1 & 4 & & 2 & & 3 & 2 & 1 & 13 & 8,8 \\
\hline $\begin{array}{l}\text { Myceliophthora vellerea (Sacc. \& Speg.) } \\
\text { van Oorshot y su teleomorfo Ctenomyces } \\
\text { serratus Eidam }\end{array}$ & 2 & 3 & 1 & & 1 & 4 & 1 & $\mathbf{3}$ & 15 & 13,4 & 2 & 4 & 2 & 2 & 1 & 2 & & 1 & 14 & $\mathbf{9 , 5}$ \\
\hline $\begin{array}{l}\text { Trichophyton terrestre Durie \& D.Frey y } \\
\text { su teleomorfo Arthroderma quadrifidum } \\
\text { Dawson \& Gentles }\end{array}$ & & 1 & & 3 & & & & & 4 & 3,6 & 2 & & 1 & 2 & & 2 & & & 7 & 4,8 \\
\hline Totales & 16 & 16 & 9 & 20 & 13 & 13 & 15 & 10 & 112 & & 23 & 18 & 18 & 29 & 7 & 21 & 22 & 9 & 147 & \\
\hline
\end{tabular}

5 y 8 en zonas urbanas (Gráfico 2, Tabla 1).

Las especies de anamorfos de Onygenales con mayor frecuencia en zona urbana, correspondieron a Keratinomyces ajelloi (26\%), Myceliophthora vellerea (13\%), Chrysosporium pannicola (12\%), Microsporum gypseum (12\%), M.fulvum (9\%), Chrysosporium keratinophilum y Chrysosporium anamorfo de Aphanoascus fulvescens (8\%). En la zona rural Keratinomyces ajelloi mantiene su dominancia, aunque en menor proporción (16\%), siguiéndole en porcentajes Chrysosporium anamorfo de A. fulvescens (12\%), C. pannicola (10\%), Myceliophthora vellerea (10\%), Microsporum gypseum 


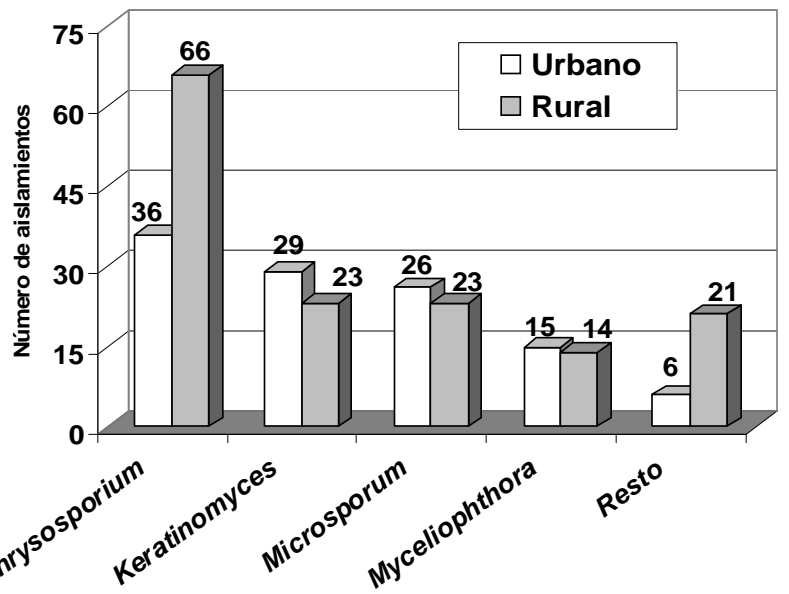

Gráfico 1.- Géneros de Onygenales aislados con mayor frecuencia en ambas zonas de muestreo.

y C. keratinophilum (9\%) (Tabla 1, Gráfico 1).

En zonas rurales se detectaron las siguientes especies: Amauroascus mutatus, Auxarthron umbrinum, Chrysosporium charmichaelii, Ch. merdarium, Ch. tropicum, Geomyces pannorum, Gymnoascus reessii, ausentes en zonas urbanas, mientras Malbranchea flava, sólo se detectó en zona urbana (Tabla 1).

Aún cuando hubo diferencias en el porcentaje (20,5\% urbano y 15\% rural), el complex Microsporum gypseum presentó la misma frecuencia de presencia en ambas zonas (Tabla 1). La diferenciación de los anamorfos integrantes del complex permitió establecer presencias semejantes de M.gypseum y M. fulvum en ambos tipos de establecimientos educacionales, aunque con mayor número de presencia del primero (Tabla 2). Todas las

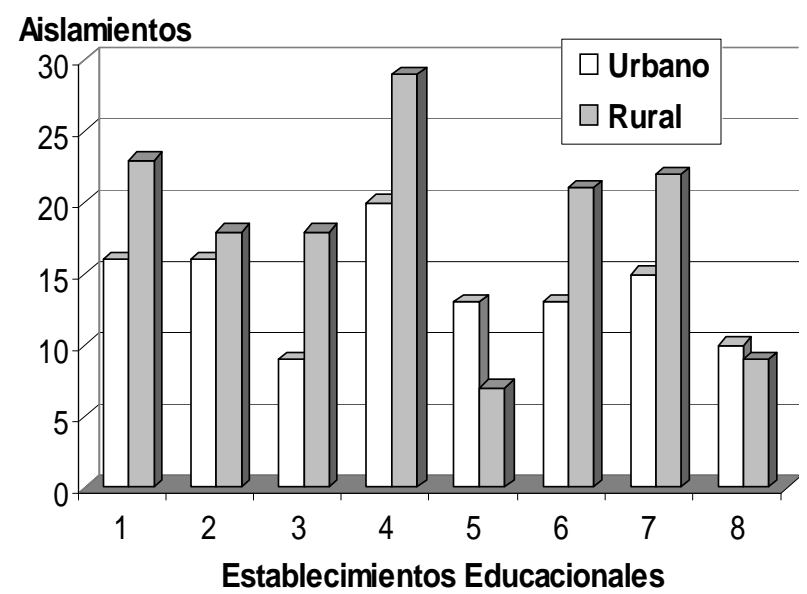

Gráfico 2. Número de aislamientos de Onygenales en los distintos establecimientos educacionales
Tabla 2: Complex Microsporum gypseum (agar Takashio) y M. cookei en zona urbana y rural

\begin{tabular}{|l|c|c|c|c|}
\hline & \multicolumn{2}{|c|}{ Urbano } & \multicolumn{2}{c|}{ Rural } \\
\hline Microsporum & $\mathrm{N}^{\circ}$ & $\%$ & $\mathrm{~N}^{\circ}$ & $\%$ \\
\hline M. gypseum & 13 & 11.60 & 13 & 8.84 \\
\hline M. fulvum & 10 & 8.92 & 9 & 6.12 \\
\hline M.cookei & 3 & 2.67 & 1 & 0.68 \\
\hline Total & $\mathbf{2 6}$ & & $\mathbf{2 3}$ & \\
\hline
\end{tabular}

especies de M. gypseum y M. fulvum se desarrollaron a $37^{\circ} \mathrm{C}$.

El índice de Shannon-Wiever mostró que la diversidad entre ambas zonas no difiere mayormente, en la zona urbana el valor fue de 2,2 y en la rural 2,6, mientras el índice de Jaccard arroja un 57\% de similitud entre ambas zonas.

\section{DISCUSION}

Los hongos queratinofílicos han generado un gran interés ecológico, particularmente para los epidemiólogos médicos y veterinarios, debido al significativo rol en la degradación natural de los residuos queratínicos y al de algunos de sus integrantes en infecciones superficiales en el hombre y en los animales (Shtayeh \& Jamous, 2000). $\mathrm{Su}$ presencia en ambientes diversos, incluso los más extremos como los antárticos (Mercantini et al., 1989; Onofri et al., 2007), mantienen patrones variables de distribución en dependencia a factores geográficos, edáficos, climáticos y la presencia humana asociada a una gran diversidad de vertebrados e insectos. Estudios sobre este grupo se han realizado en diferentes hábitat de suelos en diversos países incluyendo Chile (Ajello et al., 1965, 1974; Ajello \& Alpert, 1972; Zaror, 1972; Caretta \& Piontelli, 1975; Caretta et al., 1976; Piontelli et al., 1984 , 1990; AbdelHafez \& El-Sharouny, 1990; Marchisio et al., 1991; Ulfig et al., 1997; Toro et al., 2002; Shtayeh \& Jamous, 2000).

El uso del anzuelo queratínico como técnica de rutina selectiva para el aislamiento de este grupo especializado de hongos, al estimular su actividad fisiológica y competitiva frente a esta estable proteína animal, permitió un buen rendimiento para el objetivo de este estudio (Orr, 1969; De Vroey, 1970; Benedick, 1982).

El mayor aislamiento de los integrantes de los Onygenales en establecimientos escolares en zona rural puede asociarse al contenido orgánico de los suelos, sus ambientes rodeados de áreas verdes y parques que por la particular presencia de animales domésticos, como perros y gatos, animales de labranza y bovinos, sumada a la 
actividad de los niños en las aulas, aportan el material queratínico necesario para mantener la presencia y diversidad de estos hongos en zonas menos antropofizadas (Otcenasek et al., 1967; Dominik et al., 1973; Van Gelderen \& Elias, 1978; Marchisio, 1986; Zaror et al., 1986; Piontelli \& Toro, 1987; Caretta et al., 1976, 1989; Mercantini et al., 1995; Armes \& Hilda, 1998; Mangiaterra et al., 2000; Shadzi et al., 2002; Vidyasagar et al., 2005).

Los integrantes del género Chrysosporium obtuvieron los mayores aislamientos y el mayor número de especies en ambas zonas, pero con un notable aumento de frecuencia de presencia y diversidad en la zona rural. Este taxon guarda relacion con los dermatofitos, por tener teleomorfos en algunas especies de Artroderma y en especial en otros miembros de la familia Gymnoascaceae, como Aphanoascus, el cual se presentó muy asociado en nuestros aislamientos. Las 3 especies más frecuentes de Chrysosporium: Ch. anamorfo de Aphanoascus fulvescens, C.keratinophilum y C.pannicola, mantuvieron semejante distribución en ambas zonas, destacando el primero por su mayor presencia en zona rural. Estas especies saprófitas como otras del género, son los hongos queratinofílicos de más amplia difusión en en el suelo como en pelos de mamíferos domésticos y silvestres, plumas de aves silvestres y de crianza, pezuñas, cuernos, lana de las ovejas, nidos de pajaros, sedimentos, suelos contaminados, entre otros (Otcenasek et al., 1967; Mantovani et al., 1982; Abdel-Hafez \& Sharouny, 1990; Abdel-Hafez et al, 1990; Caretta et al., 1992; Ulfig et al., 1997; Gugnani, 2000). Han sido registradas con anterioridad en suelos chilenos en diversas latitudes geográficas y en ambientes urbanos y silvestres y consideradas, en especial las 2 primeras especies mencionadas, como euridominantes (constantes y dominantes) en todas las latitudes estudiadas (Piontelli et al., 1990). Al parecer, las especies de Chrysosporium se adaptan a diferentes tipos de suelo desde los desérticos a los ricos en humus e incluso la altitud no parece ser un factor limitante para su distribución y adaptación (Piontelli \& Caretta, 1975). La fisiología, el potencial biológico del género para reciclar desechos queratínicos u otros, la secreción de ezimas y antimicrobianos son las cualidades más sobresalientes del taxon y una nueva y actualizada cantidad de información se ha condensado al respecto en estos últimos tiempos (Kumar \& Kushwaha, 2000). Un estudio sobre la invasión de estos agentes en el pelo humano in vitro, muestra que A. fulvescens lo invade a través de la cutícula sin formar órganos perforadores especializados, mientras que en $\boldsymbol{A}$. keratinophilus la actividad queratinolítica está confinada a la corteza y se extiende posteriormente a la cutícula. $\boldsymbol{A}$. fulvescens ha sido detectado en varias ocasiones como agente etiológico de dermatomicosis en hombres y animales (Cano \& Guarro, 1990; Cano et al.1991), es considerado también como un hongo coprófilo, presente en los excrementos de herbívo-ros, donde la fuente de queratina sea probablemente el pelo ingerido en la alimentación (Cano et al., 1991). Llama la atención que $\boldsymbol{C h}$. tropicum fue aislado con baja frecuencia sólo en la zona rural, siendo descrito en la literatura como activamente queratinolítico con amplia distribución y habilidad competitiva con otros hongos queratinofílicos, por sus capacidades antagónicas que favorecen su distribución (Guarro et al., 1981; Kaul \& Sumbali, 2000).

Aunque las especies mencionadas, como otras del género, han sido aisladas con escasa frecuencia en lesiones dérmicas humanas y animales, la habilidad de permanecer viables por varias semanas en la piel $u$ otros tejidos, indica su posible oportunismoy potencial patogénico bajo ciertas circunstancias (Frey et al., 1979; Rippon, 1983; Chabasse et al., 1989; De Hoog et al., 2000; Gugnani, 2000; Kumar \& Kushwaha, 2000 ).

Keratinomyces ajelloi y su teleomorfo Arthroderma uncinatum y Myceliophthora vellerea y su teleomorfo Ctenomyces serratus, fueron los taxa keratinofilicos más aislados después de Chrysosporium, con similares proporciónes en frecuencia de presencia en zonas urbana y rural. Ambas son especies geofílicas de amplia distribución, comúnes en suelos ricos en humus, queratina, en especial plumas ( $\boldsymbol{C}$. serratus) y con $\mathrm{pH}$ alcalinos (Caretta et al., 1992), pero también asociados a mamíferos de vida libre y aves diversas, pudiendo causar el primero escasas infecciones de la piel en el hombre y los animales (Hubalek, 2000).

Las 2 especies más frecuentes del género Microsporum, M.gypseum y M. fulvum con sus teleomorfos Arthroderma gypseum y A.fulvum, son integrantes del M.gypseum complex, que incluye 4 teleomorfos distintos (Arthroderma gypseum, A. uncinatum, A f fulvum y A.corniculatum). Ambas especies, no diferenciadas normalmente en los estudios ecológicos (Hubalek, 2000), obtuvieron similares frecuencias de presencia $\left(\mathrm{N}^{\circ}\right.$ de aislamientos) en ambas zonas y en general muestran afinidad por los suelos ácidos y aquellos que reciben aguas contaminadas provenientes de las ciudades. Son especies de amplia distribución, detectadas en los areneros de niños y en los patios de los colegios y ambientes internos por diversos investigadores con resultados similares a los nuestros (Dominik et al., 1973; Van Gelderen \& Elias, 1979; Marchisio, 1986; Shtayeh 1989; Mangiaterra et al., 2000).

En un estudio realizado en el ambiente de 11 hospitales (polvo de pasillos) y 21 suelos de áreas recreacionales en India, se aislaron 41 especies entre dermatófitos y especies relacionadas, donde $\boldsymbol{M}$. gyseum fue la especie dominante, seguida por Ch. keratinophilum 
(Vidyasagar et al., 1995). M.gypseum, es una especie potencialmente patógena cuyos reportes sobre infecciones en la piel del hombre y de los animales han ido en aumento (Alvarez \& Bracalenti, 1984; Piontelli \& Toro, 1987; Connole, 1990; Marchisio et al., 1996; Porro et al., 1997; Mangiaterra et al., 2000; García-Martos et al., 2004). Por esta razón, no es extraño que nuestras cepas de sembrados e incubados a $37{ }^{\circ} \mathrm{C}$, crecieran bien en los medios utilizados, confirmando la potencialidad patógena de esta especie.

En estudios de patogenicidad en animales de laboratorio con los integrantes del complex M.gypseum, se ha observado que A.gypseum y A.incurvatum no muestran notables diferencias en patogenicidad, mientras A.fulvum es el menos patogénico (Sympanya, 2000; García -Martos et al., 2004).

La separación de las especies dentro de los anamorfos del complex M.gypseum, fueron identificadas fácilmente según la técnica expuesta por Demange et. al., (1992) y permitió reconocer la presencia en ambos ambientes de sólo 2 de sus integrantes (M.gypseum y M.fulvum). Se aconseja, como un método refinado, dejar las pruebas de cruzamientos sexuales de mayor complejidad, para las cepas atípicas, debido a la necesidad de tener cepas parentales recombinantes $(+\mathrm{y}-)$.

En referencia a los otros aislamientos obtenidos en bajas frecuencias o escasas en ambas zonas no existe consenso en relación a su rol oportunista y más bien son considerados como queratinofílicos con habilidad competitiva saprofítica.

El conocer la presencia de ciertos taxa fúngicos queratinofílicos en ambientes antropofizados urbanos como rurales o periféricos, contribuye en el esclarecimiento de posibles contagios directos o indirectos desde el suelo e induce a evaluar los conceptos de saprofitismo y parasitismo, bajo ciertas circunstancias favorables, no como estrategias eco-nutricionales separadas, sino como una posible continuidad en el contexto epidemiológico, que debería considerarse en las áreas escolares analizadas.

\section{CONCLUSIONES}

Mediante la técnica del anzuelo queratínico se detectó la presencia en los suelos de los establecimientos educacionales estudiados, de un grupo particular de hongos pertenecientes al órden de los Onygenales, considerados como queratinofílicos que sobreviven y se dispersan en este ambiente mediante la utilización de este particular subtrato aportado ya sea desde fuentes humanas como de animales de compañía o silvestres.

En el medio rural se obtuvieron los mayores aislamientos fúngicos y una mayor diversidad de especies que en el urbano.
Los géneros Chrysosporium y Microsporum obtuvieron las mayores frecuencia de presencia en ambas zonas, especialmente Chrysosporium en rural, donde presentó el mayor número de especies, destacándose $\boldsymbol{C h}$. pannicola en en zona urbana y Chysosporium anamorfo de Aphanoascus fulvescens en rural. M.gypseum y M.fulvum obtuvieron aislamientos semejantes en ambas zonas. Aún cuando todas las especies aisladas tienen habilidades competitivas saprofíticas en los suelos, debe considerarse que frente a un aumento de la densidad humana (escuelas), algunas especies de Chrysosporium y particularmente M.gypseum pueden ser responsables de algunas micosis oportunistas superficiales.

\section{REFERENCIAS}

Abdel-Hafez, A.L.L.; Moharram, A.M. \& Abdel Gawad,K.M. (1990). Survey of keratinophilic fungi in the kloven-hooves and horns of goats and sheep from Egypt. J. B. Microbiol.30:13-20

Abdel-Hafez, A.L. \& El Sharouny, H.M.M. (1990). The occurrence of keratinophilic fungi in sewage sludge from Egypt. J. Basic Microbiol. 30:73-79

Ajello, L.(1960). Geographic distribution and prevalence of dermatophytes. Ann. N.Y. Acad Sc. 89:30-38

Ajello, L.; Varsavsky, E.; Delvingt,W.(1965). Keratinophilic fungi from belgian soils. Trans. Br. Mycol. Soc. 48:417-421

Ajello, L. \& Alpert, E.M. (1972). Survey of easter island soils from keratinophilic fingi. Mycologia 64:161-166

Ajello, L. (1974). Natural history of the dermatophytes and related fungi. Mycopath. Et. Mycol. Appl. 53:93-110

Alvarez, P. \& Bracalenti, B. (1984). Aislamiento de cepas queratinolíticas con modificación del anzuelo queratínico. Bol. Micol. 2:1-4

Armes, V.M. \& Hilda, A. (1998). Incidence of keratinophilic fungi in the soils of pimary schools and public parks of Madras city, India. Mycopathologia 143:139-147

Benedick, T. (1982). Fragmenta Mycologica I. Some historial of the development of hair baiting of Toma-Karting- Vanbreuseghem (The Tokava hair baiting Method). Mycopathol. Mycol. Appl. $16: 104-106$

Cano, J. \& Guarro, J. (1990). The Genus Aphanoascus. Mycol.Res. 94:355-377

Cano, J.; Guarro, J. \& Figueras, M.J. (1991). Study of invasion of human hair in vitro by Aphanoascus spp. Mykoses 34:145-152

Caretta, G. \& Piontelli, E. (1975). Isolation of keratinophilic fungi from soil in Pavia, Italia. Sabouraudia 13:33-37

Caretta, G.; Del Frate,G.; Piontelli, E.; Todaro,F. (1976). Micoflora cheratinofila del pelo e dello sterco di mucca, del foraggio e del suolo di fattoria: Consideración sulla loro distribuziones. Riv. De Parassitologia. 37:333-361 
Caretta G.; Mancianti, F. \& Ajello, L. (1989). Dermatophytes and keratinophilic fungi in cats and dogs. Mykoses 32:620-626

Caretta, G.; Mangiarotti, A.M. \& Piontelli, E. (1992). Keratinophilic Fungi isolated from soil of italian parks in the province of Pavia. Eur. J. Epidemiol. 8:330-339

Chabasse, D.; De Gentile, L. \& Bouchara, J. Ph. (1989). Pathogenicity of some Chrysosporium species isolated in France. Mycopathologia 106:171-177

Charmichaeli, J. W. (1962). Chrysosporium and some other aleuriosporic Hyphomycetes. Can. J. Bot. 40:1137-1173

Connole, M.D.(1990). Review of animal mycoses in Australia. Mycopathologia 111:133-164

Currah, S.R. (1985). Taxonomy of the Onygenales: Arthrodermataceae, Gymnoascaceae, Myxotrichaceae and Onygenaceae. Mycotaxon 24:1-216

De Hoog, G.S. ; Guarro, J.; Gene, J. \& Figueras, M.J.(2000). Atlas of Clinical Fungi. Centraalbureau voor Schimmelcultures, Universitat Rovira i Virgili.

Demange C.; Contet-Audonneau, C.; Kombila, M. ; Miegeville, M. ; Berthonneau, M. ; De Vroey, Ch. ; Percebois G. (1992). Microsporum gypseum complex in man and animals. Journal of Medical and Veterinary Mycology. 30:301-308

De Vroey, Ch. (1970). Contribution a l'étude des dermatophytes et d'autres. Gymnoascaceae. Am.Soc.Belge. Med.Trop. 50:1-174

Díaz, M.C. ; Salamanca, L. \& Piontelli, E. (1984). Dermatofitosis, un problema del pasado, un desafío del presente. Adel. Microbiol. Enf. Infecc. 3:212-273

Dominik, T.; Ihnatowicz, A., Kopilow, H.; Mietkiewski, R. (1973). Mycoflora of sand boxes in kindergarden in Szczecie. Ekologia Polska, 153:901-923

Domsch, K.H.; Gams, W.; Anderson, T.H. (1980) Compendium of soil fungi. Vol.1, Academic Press, New York.

English, M.P. (1965). The saprophytic growth of nonkeratinophilic fungi on keratinized substrate, and a comparison with keratinophilic fungi. Trans. Brit. Mycol. Soc. 48:49-235

Frey,D.; Oldfield, R.J.; Bridger, R.C. (1979). A color atlas of pathogenic fungi. London Wolfe Medical Pupl.

García-Martos, P.; Ruiz-Aragón, J. ;García-Agudo, L.; Linares, M. (2004). Dermatofitosis por Microsporum gypseum: Descripción de 8 casos y revisión de la literatura. Rev. Iberoam. Micol. 21:147-149

Guarro, J.; Punsola, L. \& Calvo, M.A. (1981). Keratinophilic fungi from soil of Tarragona. Catalunya. Mycopat. 76:69-71

Gugnani, H.C. (2000) Non dermatophitic filamentous keratinophilic fungi and their role in human infection.In: Biology of Dermatophytes and other keratinophilic fungi (Kushwaha, R.K.S. \& Guarro, J. Eds.). Rev. Iberoamericana de Micología, Bilbao pp. 109-114

Hubalek, Z. (2000). Keratinophilic fungi associated with free- living mammals and birds. In: Biology of Dermatophytes and other keratinophilic fungi (Kushwaha, R.K.S. \& Guarro, J. Eds.). Rev. Iberoamericana de Micología, Bilbao. pp. 93-103

Kaul, S. \& Sumbali, G. (2000). Keratinophilic fungi from poultry farm soils of Jammu, India. Mycologist 14:89-91

Kumar, R. \& Kushwaha, S. (2000). The genus Chrysosporium, it physiology and biotechnological potential. In: Biology of Dermatophytes and other keratinophilic fungi (Kushwaha, R.K.S. \& Guarro, J. Eds.). Rev. Iberoamericana de Micología, Bilbao. pp. 66-76

Mangiaterra, M.; Giusiano, G.; Peluca, G.; Alonso, J.M. (2000). Geohongos queratinofílicos en áreas de recreación de jardines de infantes en Resistencia, Argentina. Bol. Micol. 15: $101-106$

Mantovani, A.; Morganti, L.; Battelli, G. ; Mantovani, A.L.; Poglayen, G. ; Tampieri, M.P. ; Vecchi, G.(1982). The role of wild animals in the ecology of dermatophytes and related fungi. Folia Parasitológica (Praha), 29:279-284

Marchisio, F.V. (1986). Keratinolitic and keratinophilic Fungi of children sandpits in the city of Turin. Mycopathologia 94:163172

Marchisio, F.V.; Curetti,D. \& Bordese, C. (1991). Keratinolytic and keratinophilic fungi in the soils of Papua, New Guinea. Mycopathologia 115:113-119

Marchisio, F.; Gallo, M.G.; Tullio, V.; Nepote S.; Piscozzi, A.; Cassinelli, C. (1995). Dermatophytes from a case of skin disease in cats and dogs in Turín, Italy. Mykoses 38:239-244

Marchisio, F.; Prevel,L. \& Tullio,V.(1996). Fungi responsable for skin mycoses in Turín, Italy. Mykoses 39:141-150

Mercantini, R.; Marsella, R \& Cervellati,M.C. (1989). Keratinophilic fungi isolated from antarctic soil. Mycopathologia $106: 47-52$

Onofri, S.; Zucconi, L. \& Tosi, S. (2007). Continental antarctic fungi. IHW- Verlag, München.

Orr, G.F.(1969). Keratinophilic Fungi isolated from soil by a modified hair bait technique. Sabouraudia 7:129-139

Otcenasek, M.; Dvorak, J.;Kunert, J. (1967). Geographic Distribution of the geophilic dermatophytes in the soil. Mycopathol. Mycol. Appl. 31:151-162

Piontelli, E.; Toro, M.A. (1987). Los animales domésticos (perros y gatos) como reservorio fúngico. Bol. Micol. 4:149-158

Piontelli, E.; Toro, M.A. \& Casanova, D. (1984). Diversitydominante and sucesión of fungal communities in soil (a beach of V.-Region, Chile) on keratine substrate, I. Bol. Micol. 2:73-89

Piontelli, E.; Toro, M.A. \& Casanova, D. (1990). Latitudinal distribution of Onygenales and related Hyphomycetes in soils of northern Chile between 18-34 ${ }^{\circ}$ South Latitude. Boletín Micológico 5:79-106

Porro, A.; Yoshioka, M.; Kaminski, S. (1997). Diseminated dermatophytosis caused by Microsporum gypseum in two patients 
with the acquired inmunodeficiency sindrome. Mycopath. 137:912

Rebell,G. \& Taplin, D.(1970). Dermatophytes their recognition and identification. University of Miami Press, Florida.

Rippon J.W. (1983). Clinical aspects of Medically important conidial fungi. En Cole, G.T.,Kendrick E.B. Ed. Biology of conidial fungi. Academic Press, New York. Pp: 3-5

Shadzi, S.; Chadeganipour, M. \& Alimoradi, M. (2002). Isolation of keratinophilic fungi from elementary schools and public parks in Isfahan, Irán. Mykoses 45:496-499

Shtayeh, A.(1989). Keratinophilic fungi of schoolplaygrounds in the Nablus area. West Bank of Jordan. Mycopathlogia 106:103108

Shtayeh, A. \& Jamous, R.M.F.(2000). Keratinophilic fungi and related dermatophytes in polluted soil and water habitats.In: Biology of Dermatophytes and other keratinophilic fungi (Kushwaha, R.K.S. \& Guarro, J. Eds.). Rev. Iberoamericana de Micología, Bilbao. pp. 51-59

Simpanya, M.F. (2000). Dermatophytes: Their Taxonomy ecology and Pathogenicity. In: Biology of Dermatophytes and other keratinophilic fungi (Kushwaha, R.K.S. \& Guarro, J. Eds.). Rev. Iberoamericana de Micología, Bilbao. pp.1-12

Takashio, M. (1973). Etude des phénomènes de reproduction lié au viellissement et au rajeunissement des cultures des champignons. Ann. Soc. Belg. Med. Trop. 53:427-580
Toro, M.A.; Piontelli, E. \& Casanova, D. (2002). Geohongos en ambientes acuáticos: Onygenales queratinofílicos aislados en la interfase y sedimentos del río Aconcagua, V Región (Chile). Bol. Micol. 17:21-32

Ulfig, K.; Guarro, J.; Cano, J.; Gene, J.; Vidal, P.; Figeras, M.J.; Lukasik, W. (1997). The occurrence of keratinolytic fungi in sediments of the river Tordera (Spain), FEMS. Microbiology Ecol. 22:111-117

Vanbreuseghem, R. (1952). Technique biologique pour l'isolement des dermatophytes du sol. Ann.Soc.Belg.Trop. 32:173-178

Van Gelderen, A. de K. \& Elías, F. (1978). Presencia de dermatofitos en suelos de escuelas de la provincia de Tucumán, República Argentina. Rev. Latinoam. Microbiol. 20:95-98

Van Oorschot, C.A.N. (1980). A revision of Chrysosporium and allied genera. Studies in Mycology $\mathrm{n}^{\circ} 20$. Centraalbureau voor Schimmelcultures, Baarn.

Vidyasagar, G. M.; Hosmani, N. \& Skivkumar, D. (2005). Keratinophilic Fungi isolated from hospital dust and soils of public places at Gulbarga, India. Mycopathologia 1:13-21

Zaror, L. (1972). Dermatofitos en suelos de Chile. Estudio preliminar. Bol. Inst. Bact. de Chile. 14:31-35

Zaror, L.; Fischmann, O.; Borges, M.; Vilanova, A.; Levites, J. (1986). The role of the cats and dogs in the epidemiological cycle of Microsporum canis. Mykosen 29:185-188 